\title{
Computer-Aided Optimization of Multilayer OLED Devices
}

\author{
Franz Symalla*, Pascal Friederich ${ }^{* *}$, Simon Kaiser ${ }^{\star *}$, Timo Strunk$^{*}$, Tobias Neumann \\ Wolfgang Wenzel ${ }^{* *}$ \\ *Nanomatch GmbH, Eggenstein-Leopoldshafen, Germany \\ **Karlsruhe Institute of Technology, Karlsruhe, Germany
}

\begin{abstract}
Development of efficient OLED devices is presently driven by experimental trial\&error $R \& D$. We developed a bottom-up multiscale modeling approach enabling the computation of device properties without the use of experimentally determined parameters. Researchers can identify bottlenecks, develop new materials and optimize devices using computer aided design.
\end{abstract}

\section{Author Keywords}

OLED stack design - computer aided design - material development - multiscale modeling - predictive simulations

\section{Introduction}

While computer simulations are widely used in organic electronics to support experimental R\&D, established methods such as drift diffusion are based on parametric models and simulations rely on experimental input for parameters such as energy levels or charge carrier mobilities. This prevents the design of novel materials and optimization of devices fully in the computer. Therefore, industrial R\&D relies on time consuming and costly trial and error approaches.

We overcome this barrier with a bottom-up multiscale modeling approach starting on the quantum mechanical level that maps single molecule properties to the device scale without the use of external parameters, e. g. from experiment. By including relevant effects and processes on the microscopic scale, this approach (i) generates insight on information that is not accessible by experiments (artificial microscope) and (ii) allows researchers to analyze the impact of specific microscopic effects on device performance. This enables a straightforward and systematic identification of bottlenecks and allows the targeted development of compounds tailored for specific purposes and the optimization of layer architectures, fully in the computer.

\section{Methods}

In order to compute properties on the device level, we construct a digital twin of the device in the computer. This is done in four simulation steps:

1. Single molecule parametrization:

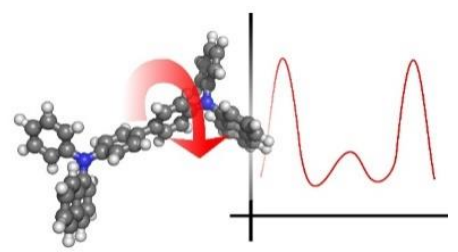

Geometries and electronic properties of single molecules (the constituents of e. g. HTL or ETL, host materials, dopants) are optimized using quantum mechanical ab initio methods. Customized force-fields describing inter and intra-molecular interactions are generated automatically for each molecule for the simulation of the thin film formation.

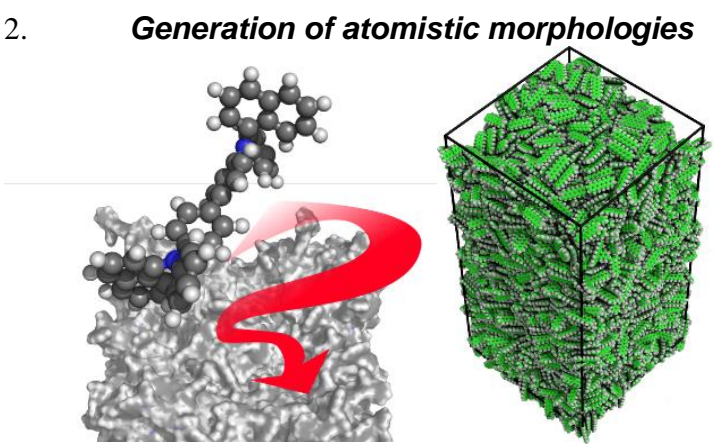

Based on the customized force-fields, molecular thin-films with atomic resolution are generated. Parallelized, grid-based forcefield evaluation allows the fast deposition of morphologies on the $10 \mathrm{~nm}$ scale. By mimicking physical vapor deposition, experimentally observed features, such as inhomogeneities and anisotropies are exhibited in these structures. [1]

\section{Electronic structure analysis:}

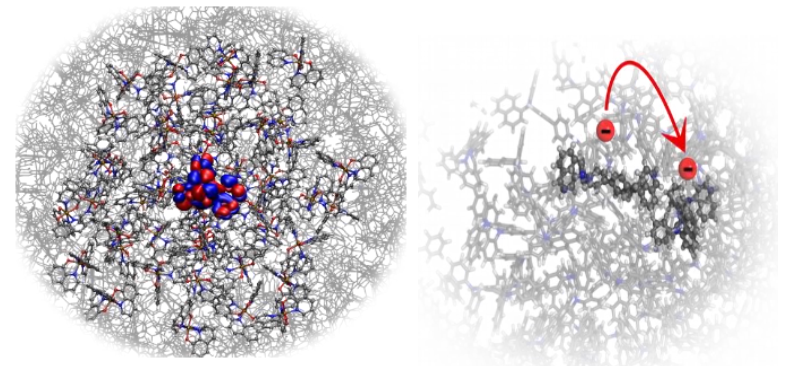

Atomistic morphologies are then analyzed on full quantum mechanical level. Electronic information necessary to compute charge and energy transfer rates (such as local energy levels or intermolecular electronic coupling) is calculated. Environmental effects are taken into account exclusively on quantum mechanical level, enabling the analysis of pristine layers, doped systems and specific interface effects. Distributions of electronic properties on the $10 \mathrm{~nm}$ scale are mapped on the device scale to enable full device simulation. [2-7]

\section{Computation of hopping rates and charge transport simulations:}

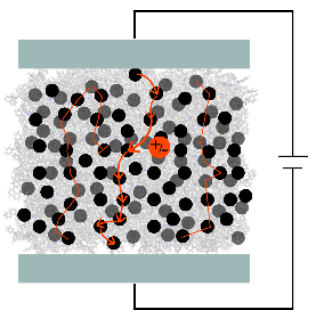

Based on the electronic structure in the molecular layers, charge transport on the device level, from single layers to multi-stack devices, is calculated using a kinetic Monte-Carlo approach. Rates for the individual processes are computed according to Marcus theory based on the electronic structure 
calculations of step 3. [8] Taking into account relevant excitonic and charge transport processes, these simulations allow the detailed analysis of microscopic bottlenecks in device performance.

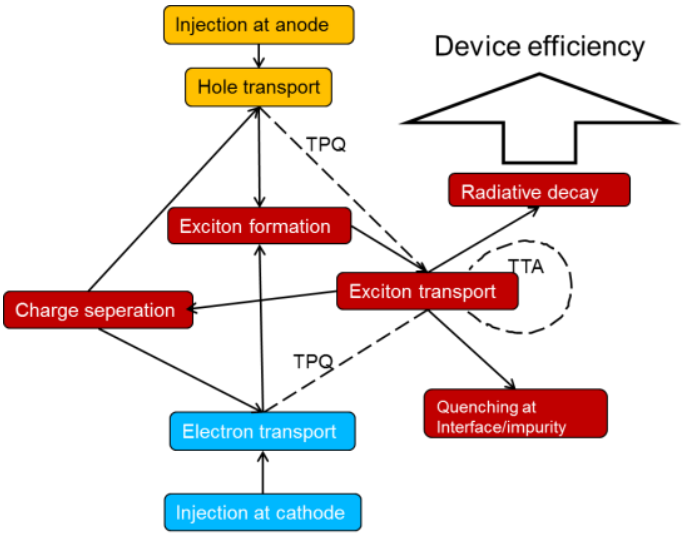

Figure 1: Microscopic processes modeled in OLED device simulations using a kinetic Monte Carlo (kMC) protocol as implemented in LightForge kMC.

Processes modeled in the device simulations are displayed in Fig. 1. At the electrodes, charges (electrons or holes) are injected. acoording to rates reflecting the local Coulomb potential and electronic energy levels. Charges can then propagate in the device, according to the ab-initio based hopping rates. Energy levels and hopping rates are continously reevaluted accouting for changes in the local coulomb potential. Recombination and exciton separation rates for electrons and holes are calculated based on local ionization energy, local electron affinity, local coulomb potential, optical gap and intermolecular coupling and spacing. This means changes of e.g. interface charge densities during operation will influence recombination rates. Once formed, excitons diffuse and decay according to specified Förster radii, radiative lifetimes and ab-initio based rates for Dexter transfer. Triplet-triplet annihilation is treated heuristically, such that a transfer of an exciton to an excited molecule will quench the original exciton. Triplet-polaron quenching is treated equivalently: energy transfer on a charged molecule quenches the exciton, as does charge transfer on an exciton.

\section{Results}

\section{Bulk mobilities of host and transport materials}

To validate this approach we computed charge carrier mobilities in pristine layers of compounds widely used as host materials in OLED emissive layers or in transport layers: NPB $\left(\mathrm{N}, \mathrm{N}^{4}\right.$ di (naphthalen-2-yl) -N,N'-diphenyl-benzidine), TCTA (4, $44^{\prime}, 4^{\prime \prime}$ tris (N-carbazolyl) -triphenylamine), and C60. For C60, zero field mobilities were calculated by extrapolating the field dependence from the KMC simulations to zero field. Results are displayed in Fig. 2, in comparison with experimental data. Field dependent data is included, where available. Mobilities for NBP and TCTA show excellent agreement with experimental data for all simulated fields. Zero field mobilities, that can be used as input for e.g. drift diffusion simulations, show a deviation of less than a factor 2 for the tested materials while spanning three orders of magnitude. We note that no parameters were tuned in this approach as the computation relies solely on quantum mechanical information derived with DFT methods.

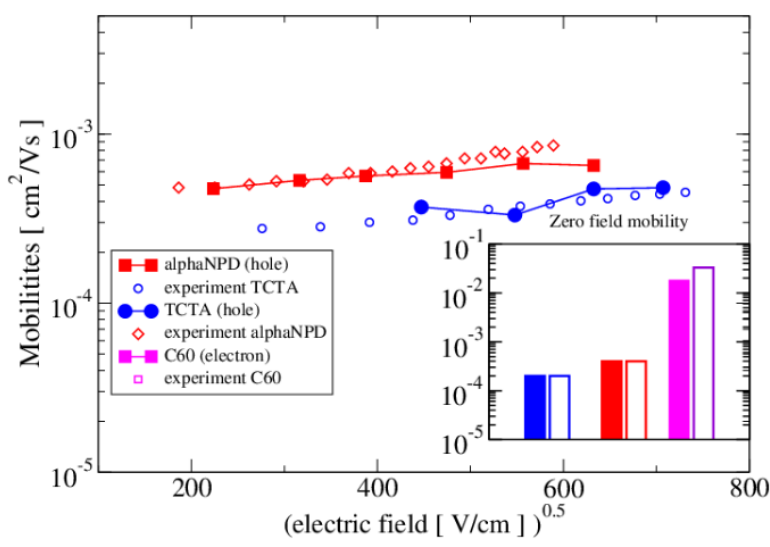

Figure 2: Comparison of experimental and computed field dependency of charge carrier mobilities for NBP and TCTA, as well as zero field mobilities for NBP, TCTA and C60 (inlet). Zero field mobilities were computed by extrapolating the field dependence in KMC simulations. Experimental values are taken from [9] for C60, [10] for NBP and [11] for TCTA.

\section{Stack design from scratch}

To demonstrate how the OLED workflow can be applied to design OLED stacks from scratch, solely on the basis of single molecule information, we constructed a minimal working example on the basis of the molecule ADP $(9,10-$ Diphenylanthracene). To construct an interface for exciton generation, i.e. a shift in energy levels, we introduce a second layer consisting of ADP, in which we substituted two carbon atoms for nitrogen. The device setup is displayer in Fig. 3.

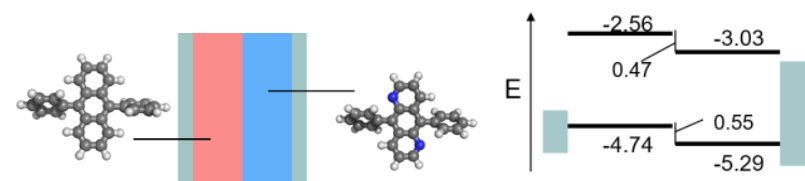

Figure 3: Left: Setup of the minimal working example for OLED design from scratch: One layer consists of ADP, the other layer of ADP where we substituted two carbon atoms for nitrogen. This shifts the energy level to create an interface for exciton generation. Right: Computed energy levels of the bilayer system.

Following the OLED workflow described in Sec. 2, we generated atomistic morphologies and computed energy levels (Fig. 3, right), energy disorder and electronic couplings for pristine layers of ADP and $\mathrm{ADP}(\mathrm{N})$ as well as for an interface of both materials. Using these results, microscopic rates for charge transport were computed and applied in a KMC simulation to compute field dependent current (I-V characteristics) and internal quantum efficiency (IQE, roll-off). IQE was computed by counting photons emitted and dividing by number of charges injected into the system at the electrodes over the course of the simulation. I$\mathrm{V}$ and roll-off are displayed in the top panel Fig. 4. To investigate the reason for the roll-off, spatial distributions of charge carriers, exciton generation, photon emission and exciton quenching were analyzed at two different voltages in the bottom panel of Fig. 4. 


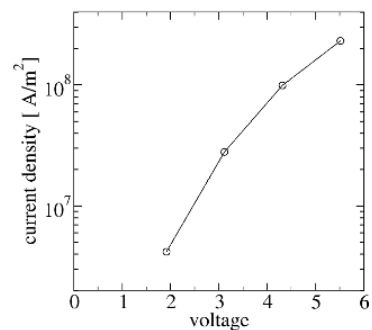

$2.0 \mathrm{~V}$

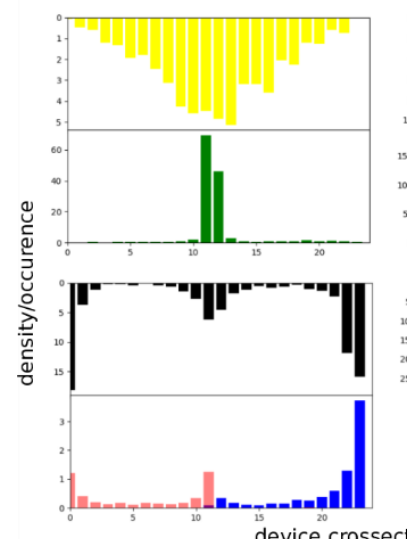

device crossection / $\mathrm{nm}$

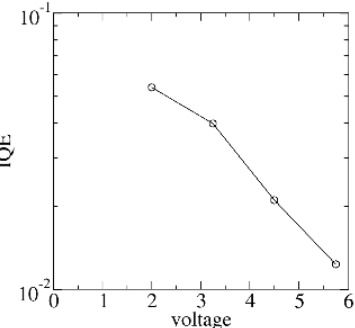

$5.75 \mathrm{~V}$
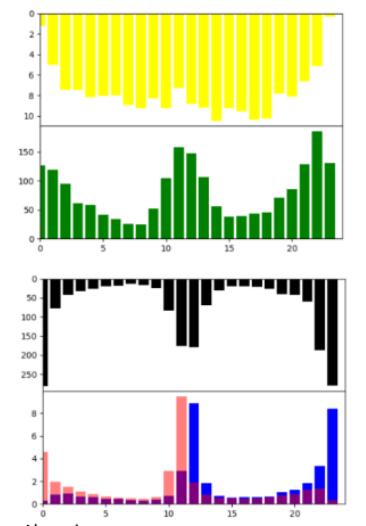

Figure 4: Top: I-V (left) and roll-off (voltage dependent IQE, right) of the bilayer stack. Bottom: Photon generation (yellow), exciton generation (green), exciton quenching (black) and charge carrier distribution/density throughout the device, for $\mathrm{U}=2.0 \mathrm{~V}$ and $\mathrm{U}=5.75 \mathrm{~V}$. At higher voltages, charge density at the interface is higher and the interface efficacy in blocking holes and electrons is reduced, leading to an increase in exciton quenching processes with electrons or holes as well as higher leakage current, and therefore a decrease in device efficiency (=roll-off).

The KMC simulations, solely based on quantum mechanical information, provide a smooth I-V curve, as well as the drop in IQE for higher voltages (roll-off) that is typically observed in OLED devices. The fundamental reason for the roll-off can be explained by the distributions in the bottom panel of Fig. 4: At low voltages, excitons are created mostly in the interface region, while charge at the interface can be depleted. At higher voltages, however, the increased interface charge cannot be depleted quick enough, leading both to higher charge concentration and penetration of the blocking layer. The generally higher charge density in areas where excitons are formed lead to exciton quenching. Due to the penetration of the interface, there is significant exciton generation also within the layers and at the electrodes, i.e. in regions of high charge density. The overlap of charge density and exciton creation density leads to an increased number of exciton quenching processes, thereby decreasing IQE. Furthermore, at high voltages, single charge carriers reach the opposite electrodes without generating excitons.

Starting from the initial idea of a simple bilayer OLED device, the conducted simulations give clear strategies for device improvement: (1) blocking layers prevent charges to travel beyond the emissive zone and (2) emissive layers with guest-host systems can be introduced to confine excitons to a defined emissive zone, minimizing quenching processes and therefore improving roll-off.

While these are in fact no novel insight in OLED design, this exemplifies how parameter-free computer simulations can be applied to iteratively design OLED devices bottom-up starting on the molecular level. The workflow is easily extended to state-ofthe-art multilayer stacks, to either screen device parameters such as compounds, guest-host-combinations, doping, layer thicknesses, etc., or to systematically gain insight on microscopic processes that is hard to access in experiment (or not at all) and determine fundamental bottlenecks in device performance.

\section{Discussion}

In this work we presented a seamless workflow for the ab-initio computation of OLED device properties. In contrast to other methods, no parametrization e.g. from experiment is required, enabling full virtual design of materials and devices. The approach was validated against experimental data for charge carrier mobilities in host materials/transport layers, showing excellent agreement between simulation and experiment. To demonstrate the workflow as a tool to stack design from scratch, we applied it to a bilayer system and analyzed microscopic processes to identify the bottlenecks in device performance and derive strategies for efficiency improvement.

\section{Acknowledgements}

This work was partially funded by the EU H2020 project EXTMOS (grant no. 646176).

\section{References}

[1] T.Neumann et al., "Modeling disordered morphologies in organic semiconductors", J. Comput. Chem. 2013, 34, 27162725,2013

[2] P.Friederich et al., „Ab initio treatment of disorder effects in amorphous organic materials: Toward parameter free materials simulation" J. Chem. Theory Comput., 2014, 10 (9), Pages 3720-3725

[3] P.Friederich et al., „QM/QM approach to model energy disorder in amorphous organic semiconductors" J. Chem. Theory Comput., 2015, 11 (2), Pages 560-567

[4] P. Friederich et al., "Multiscale Simulation of Organic Electronics Via Smart Scheduling of Quantum Mechanics Computations" Procedia Comput. Sci., 2016, 80, Pages 12441254

[5] P.Friedrich et al., „Molecular origin of the charge carrier mobility in small molecule organic semiconductors" Adv. Functional Mater. 2016, 26 (31), Pages 5757-5763

[6] P. Friederich et al., "Rational In Silico Design of an Organic Semiconductor with Improved Electron Mobility" Adv. Mater. 2017, 29, 1703505.

[7] P. Friederich et al., "Al-Komplex-Verbindungen." Patent application EU 102017207 327.6.

[8] F.Symalla et al., "Charge transport by superexchange in molecular host-guest systems" Phys. Rev. Lett. 117, 276803 (2016)

[9] Pandey et al., "Efficient organic photovoltaic cells based on nanocrystalline mixtures of boron subphthalocyanine chloride and C60" Adv. Funct. Mater. 22, 617-624 (2012)

[10] Noh et al., "Carrier conduction mechanism for phosphorescent material doped organic semiconductor" J. Appl. Phys. 105, 033709 (2009)

[11] Matushima et al., Thin Solid Films, Vol.520.6, Pages 22832288 (2012) 